\title{
Anisotropic Rabi model
}

\author{
Qiong-Tao Xie, ${ }^{1,2}$ Shuai Cui, ${ }^{1}$ Jun-Peng Cao, ${ }^{1,3}$ Luigi Amico, ${ }^{4,5,6, *}$ and Heng Fan ${ }^{1,3, \dagger}$ \\ ${ }^{1}$ Beijing National Laboratory for Condensed Matter Physics and Institute of Physics, \\ Chinese Academy of Sciences, Beijing 100190, China \\ ${ }^{2}$ School of Physics and Electronic Engineering, Hainan Normal University, Haikou 571158, China \\ ${ }^{3}$ Collaborative Innovation Center of Quantum Matter, Beijing 100190, China \\ ${ }^{4}$ CNR-MATIS-IMM and Dipartimento di Fisica e Astronomia, Universitá Catania, \\ Via S. Soa 64, 95127 Catania, Italy \\ ${ }^{5}$ Center for Quantum Technologies, National University of Singapore, \\ 3 Science Drive 2, 117543, Singapore \\ ${ }^{6}$ Institute of Advanced Studies, Nanyang Technological University, 1 Nanyang Walk 637616, Singapore
}

(Received 8 February 2014; revised manuscript received 14 May 2014; published 11 June 2014)

We define the anisotropic Rabi model as the generalization of the spin-boson Rabi model: The Hamiltonian system breaks the parity symmetry; the rotating and counterrotating interactions are governed by two different coupling constants; a further parameter introduces a phase factor in the counterrotating terms. The exact energy spectrum and eigenstates of the generalized model are worked out. The solution is obtained as an elaboration of a recently proposed method for the isotropic limit of the model. In this way, we provide a long-sought solution of a cascade of models with immediate relevance in different physical fields, including (i) quantum optics, a two-level atom in single-mode cross-electric and magnetic fields; (ii) solid-state physics, electrons in semiconductors with Rashba and Dresselhaus spin-orbit coupling; and (iii) mesoscopic physics, Josephson-junction flux-qubit quantum circuits.

DOI: 10.1103/PhysRevX.4.021046

\section{INTRODUCTION}

There are very simple settings in physics whose understanding has very far reaching implications. This one is the case of the Rabi-type models that are possibly the simplest "organisms" describing the interaction between a spin-half degree of freedom with a single boson. Originally formulated in quantum optics to describe the atom-field interaction [1], such a kind of model plays a crucial role in many other fields, especially with the advent of the quantum technologies. Here, we introduce an anisotropic generalization of the Rabi model and discuss the exact energies and eigenstates of it. In this way, we provide a long-sought solution of a cascade of models with immediate relevance in various fields.

The Rabi-type models provide the paradigm for key applications in a variety of different physical contexts, including quantum optics [2] and solid-state and mesoscopic physics [3]. Despite its importance, such models remained intractable with exact means for many years. Nevertheless, the physical community could thoroughly

\footnotetext{
*lamico@dmfci.unict.it

†hfan@iphy.ac.cn
}

Published by the American Physical Society under the terms of the Creative Commons Attribution 3.0 License. Further distribution of this work must maintain attribution to the author(s) and the published article's title, journal citation, and DOI.
Subject Areas: Quantum Physics, Statistical Physics

analyze the Rabi-model physics, essentially because the physical settings allowed physicists to easily adjust the field frequency to resonate with the atomic bandwidth. In this way, assuming as well that the field intensity is weak, the Rabi model could be drastically simplified to the Jaynes-Cummings (JC) model [4], through the celebrated "rotating-wave" approximation. The situation radically changed in the last decade, when quantum technology has been advancing toward more and more realistic applications [5-7]. In most of the cases, if not all, the rotating-wave approximation cannot be applied. In the solid-state applications, for example, the electric field is an intrinsic quantity that cannot be adjusted. On the other hand, in the applications in mesoscopic physics (like superconducting or QED circuits), the most interesting regimes correspond to a very strong coupling between the spin variable and the bosonic degree of freedom.

The class of the anisotropic Rabi model we consider in the present paper is described by the following Hamiltonian:

$$
\begin{aligned}
H & =\omega a^{\dagger} a+\epsilon \sigma_{x}+\Delta \sigma_{z}+g\left(H_{r}+\lambda H_{\mathrm{cr}}\right), \\
H_{r} & =\left(a^{\dagger} \sigma^{-}+a \sigma^{+}\right), \\
H_{\mathrm{cr}} & =e^{i \theta} a^{\dagger} \sigma^{+}+e^{-i \theta} a \sigma^{-} .
\end{aligned}
$$

Here, $a^{\dagger}$ and $a$ are the creation and annihilation operators for a bosonic mode of frequency $\omega, \sigma^{ \pm}=\left(\sigma_{x} \pm i \sigma_{y}\right) / 2$, 
$\sigma_{x, y, z}$ are Pauli matrices for a two-level system, $2 \Delta$ is the energy difference between the two levels, and $g$ denotes the coupling strength of the rotating-wave interaction $a^{\dagger} \sigma^{-}+a \sigma^{+}$between the two-level system and the bosonic mode. For simplicity, we already take the unit of $\hbar=1$. In the Hamiltonian (1), the relative weight between the rotating and counterrotating terms, denoted, respectively, as $H_{c}$ and $H_{\mathrm{cr}}$, can be adjusted by tuning the parameter $\lambda$. When $\epsilon=0$, the Hamiltonian enjoys a discrete $Z_{2}$ symmetry, meaning that the parity of bosonic and spin excitations is conserved.

Several attempts at solving these types of model were tried through employing the Bethe-ansatz and quantum inverse-scattering techniques $[8,9]$. The isotropic Rabi model corresponding to $\theta=0$ and $\lambda=1$ was solved exactly in a seminal paper by Braak [10]. Such an achievement has allowed us to explore the physics of the Rabi model in full generality.

In this article, we present the exact solution of the anisotropic Rabi models [Eq. (1)]. We discuss how the models can be applied to important physical settings in quantum optics and in mesoscopic and solid-state physics. We also observe that such a model can be realized with cold atoms with arbitrary spin-orbit couplings.

\section{EXACT ANALYSIS OF THE SPECTRAL PROBLEM}

To focus on the main results, we first provide a schematic of the exact solution of the spectral problem

$$
H|\Psi\rangle=E|\Psi\rangle,
$$

while leaving the details in the Appendixes.

Our approach elaborates on the method originally developed by Braak [10]. In order to find a concise solution, we perform a unitary transformation on the spin degree of freedom in the Hamiltonian (1). The eigenvalues can be found as

$$
E_{n}=x_{n}-\frac{\lambda g^{2}}{\omega}
$$

where $x_{n}$ include regular and exceptional solutions. The regular solutions are solely $0 \mathrm{~s}$ of the transcendental function, while the exceptional solutions are both the $0 \mathrm{~s}$ and poles leading to a finite transcendental function and energy degeneracy. The transcendental function is as follows:

$$
G_{\epsilon}(x)=\phi_{1} \bar{\phi}_{2}-\phi_{2} \bar{\phi}_{1},
$$

where $\phi_{1}(z)=\exp (-(\sqrt{\lambda} g \xi) /(\omega) z) \sum_{n=0}^{\infty} L_{n}^{+}\left(z+\left(\sqrt{\lambda} g \xi^{*}\right) /\right.$ $(\omega))^{n}, \phi_{2}(z)=\exp (-(\sqrt{\lambda} g \xi) /(\omega) z) \sum_{n=0}^{\infty} K_{n}^{+}\left(z+\left(\sqrt{\lambda} g \xi^{*}\right) /\right.$ $(\omega))^{n}$ and $\phi_{1}(-z)=\bar{\phi}_{1}(z), \phi_{2}(-z)=\bar{\phi}_{2}(z)$. Figures 1 and 2 display the actual behavior of $G_{\epsilon}(x)$ in different

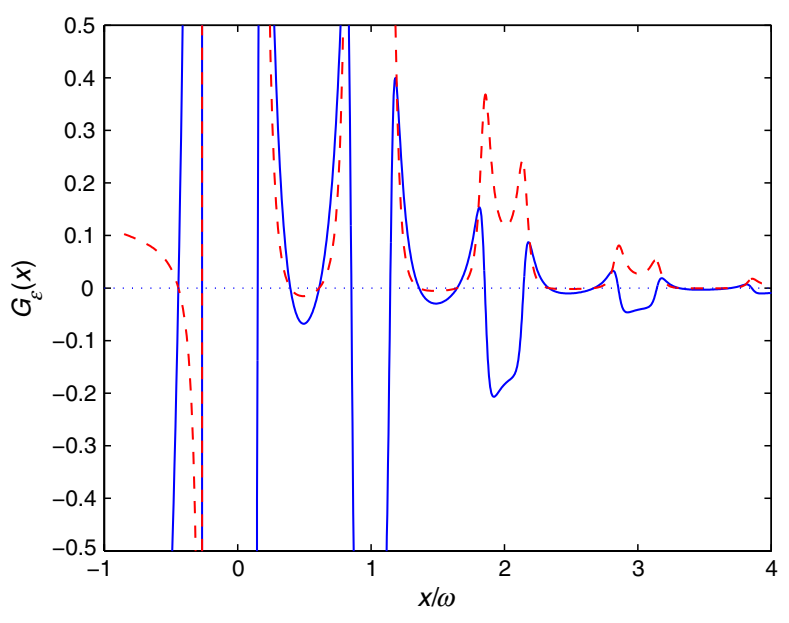

FIG. 1. Transcendental function $G_{\epsilon}(x)$ for $\epsilon \neq 0$. The parameters are $\omega=1, g=0.1, \lambda=0.5, \Delta=0.4, \quad \epsilon=0.2$, and $\theta=-\pi / 2$; the zero points whose real (solid blue line) and imaginary parts (dashed red line) of $G_{\epsilon}$ both equal 0 correspond to the eigenvalues of Hamiltonian.

parameter regimes. For $\epsilon=0$, the $Z_{2}$ symmetry is recovered; in this case, the transcendental function can be discussed through the functions $G_{+}=-e^{i \theta / 2} \phi_{1}+\sqrt{\lambda} \phi_{2}$ and $G_{-}=e^{-i \theta / 2} \phi_{2}+\sqrt{\lambda} \phi_{1}$, living separately in the two parity sectors (see Fig. 2). The explicit form of eigenfunctions $\phi_{1,2}(z)$ can also be obtained.

For vanishing $\epsilon$ or a multiple of $\omega / 2$, the system enjoys a $Z_{2}$ (parity) symmetry. In this case, the energy spectrum can

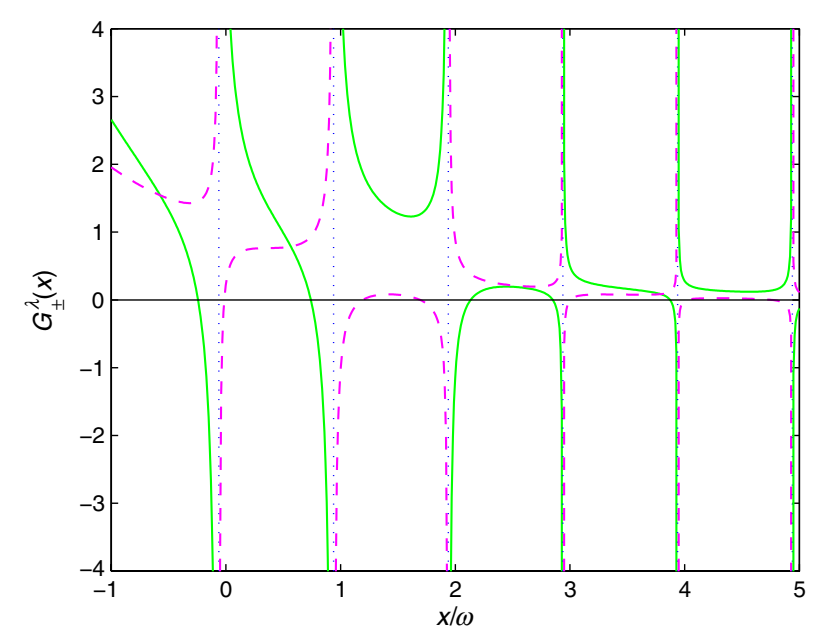

FIG. 2. Transcendental functions $G_{+}^{\lambda}(x)$ (solid green line) and real part of $G_{-}^{\lambda}(x)$ (dashed purple line) for $\epsilon=0$. The parameters are $\omega=1, g=0.7, \Delta=0.4, \lambda=0.5$, and $\theta=-\pi / 2$. The regular parts of the energy spectrum are determined by the $0 \mathrm{~s}$ of the transcendental function $G_{ \pm}^{\lambda}(x)$, and the dotted vertical lines denote the poles $x \approx n-0.0612, n=0,1,2, \ldots$; see Eq. (B13). Notice that the imaginary part of $G_{-}^{\lambda}(x)$ gives the same 0 and poles as the real part, which is not shown. 

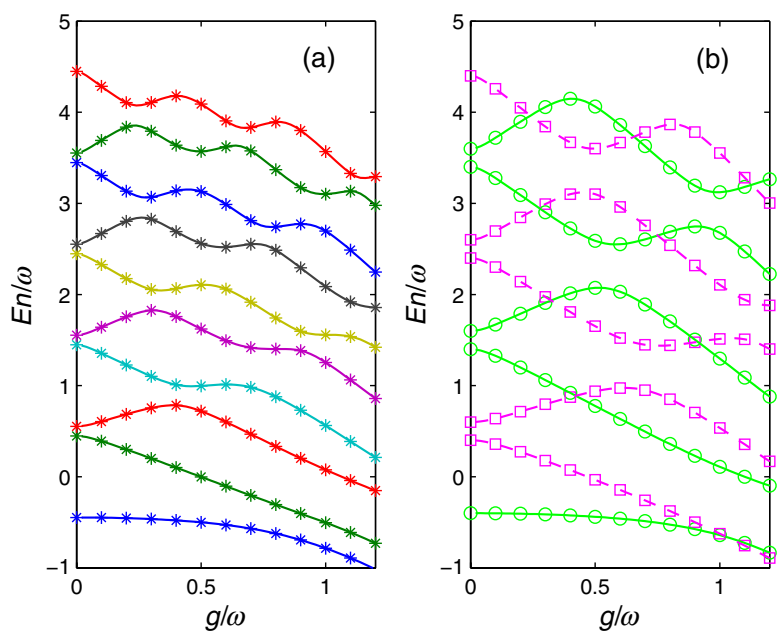

FIG. 3. Comparison between the exact solution and the numerical results. (a) Energy spectrum for $\omega=1, \Delta=0.4$, $\lambda=0.5, \epsilon=0.2$, and $\theta=-\pi / 2$. Solid lines are exact results, and the energy levels are differentiated by colors. Numerical results are represented by stars. (b) Energy spectrum for $\omega=1$, $\Delta=0.4, \lambda=0.5, \epsilon=0$, and $\theta=0$ in the spaces with positive (green lines with circles) and negative (purple lines with squares) parities. Small squares and circles represent numerical results. The first energy-level-crossing point is at $g_{c}=4 / \sqrt{15} \approx 1.0328$ and $E=-2 / 3$, which has no definite parity.

be labeled by the two eigenvalues of the parity operator [corresponding to green-with-circle and purple-with-square lines in Fig. 3(b)]. At the points of level crossings, the energy is doubly degenerate. For the isotropic case, those solutions were found previously by Braak and Judd $[10,11]$. For our anisotropic Rabi model, the crossing points are found as $E_{n}=n \omega-\left(1+\lambda^{2}\right)^{2} / 2 \omega$, corresponding to the exceptional spectrum. This exceptional spectrum is characterized by the merging of a pole with a 0 , resulting in a finite, nonzero transcendental function in Eq. (4) at energies corresponding to Juddian solutions [11]; see also Appendix B.

For nonvanishing generic values of $\epsilon$, the $Z_{2}$ symmetry is lost. This symmetry breaking is manifested in the spectrum; in particular, there are no degeneracies [see Fig. 3(a)]. As we shall see, the parameter $\theta$ is important to capturing general spin-orbit couplings. We remark that with $Z_{2}$ symmetry preserved, $\theta$ can be deleted by a unitary transformation, and thus it does not change the energy spectrum. When $Z_{2}$ symmetry is broken with nonvanishing $\epsilon$, the parameter $\theta$ enters the energy spectrum through $\epsilon \sigma_{x}$, and this unitary transformation will induce the term $\sigma_{x} \rightarrow$ $\cos (\theta / 2) \sigma_{x}+\sin (\theta / 2) \sigma_{y}$.

As it will later be argued to be important for many applications, we quantify the energy correction due to the counterrotating term (the Bloch-Siegert shift [12]). Based on the exact solution, we can give closed expressions in several interesting limits. For $2 \Delta \approx \omega, g \ll \omega$, the shift is $g^{2} / \omega$. For $\epsilon=0$, at the degenerate points, and setting $|\Delta|=\left(1-\lambda^{2}\right) g^{2} / 2 \omega$, the ground-state energy gap between the JC model and the anisotropic Rabi model can be found as

$$
\Delta E_{0}=\frac{\lambda^{2} g^{2}}{\omega}
$$

For $\lambda=1$, it is just the standard Bloch-Siegert shift $[12,13]$. Such a gap can also be obtained for the excitations. For the first and the second excited states at degenerate points, it reads approximately $\lambda^{2} g^{4} / \omega^{3}$.

\section{APPLICATIONS}

In this section, we discuss how our solution can contribute to approaching important problems in different physical contexts. Specifically, we will consider applications in quantum optics, mesoscopic physics, and spintronics.

\section{A. Application to quantum optics: Two-level atom in a cross-electric and magnetic field}

When an atom is a subject of a crossed electric and magnetic field, the selection rules are not dictated by the possible values of the atomic angular momentum. Therefore, both the electric dipole and magnetic dipole transitions are allowed. The Hamiltonian describing the system is

$$
H=H_{0}-\mathbf{d} \cdot \mathbf{E}-\mu \cdot \mathbf{B},
$$

where we have assumed that the quadrupole transitions can be neglected. Inserting the standard expressions of the quantized electric and magnetic fields are, respectively, $E \sim\left(a+a^{\dagger}\right)$ and $B \sim i\left(a-a^{\dagger}\right)$; Eq. (6) can be recast into our anisotropic Rabi model [Eq. (1)] with

$$
\begin{aligned}
& g=\frac{\langle+|d|-\rangle+\langle+|\mu|-\rangle}{2}, \\
& \lambda=\frac{\langle+|d|-\rangle-\langle+|\mu|-\rangle}{\langle+|d|-\rangle+\langle+|\mu|-\rangle},
\end{aligned}
$$

with $H_{0}| \pm\rangle=E_{ \pm}| \pm\rangle$.

\section{B. Application to superconducting circuits}

Superconducting circuits exploit the inherent coherence of superconductors for a variety of technological applications, including quantum computation [14]. In this case, the bosonic fields typically represent the electromagnetic fields generated by the superconducting currents. The spin degree of freedom describes the two states of the qubit.

As an immediate application, we consider two inductively coupled dc superconducting quantum interference devices (SQUIDs) $[15,16]$ : A primary SQUID $p$ (assumed 


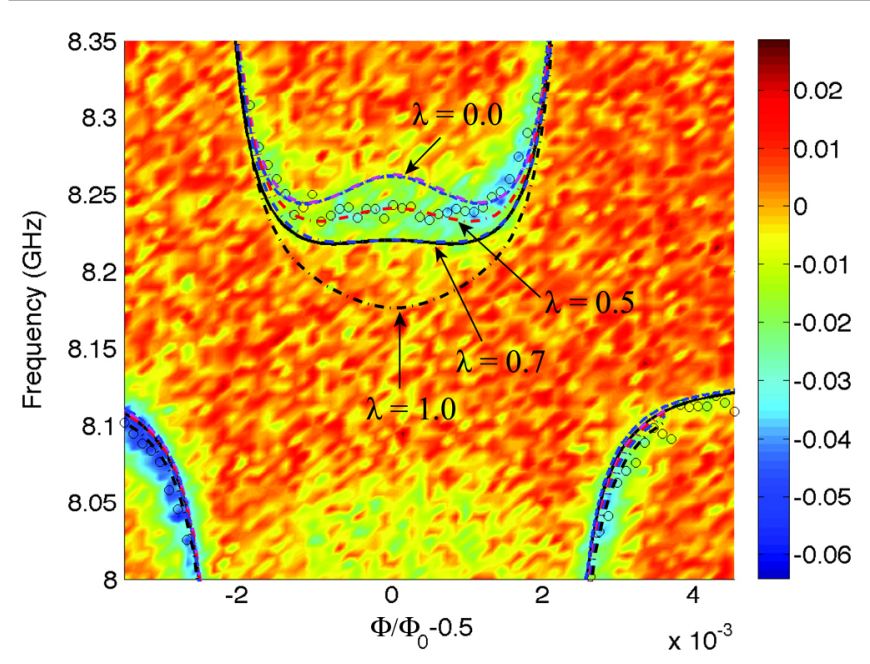

FIG. 4. Comparison between the anisotropic Rabi model with experimental results. The spectra (dot-dashed lines) of the anisotropic Rabi model with $\lambda=0.0,0.5,0.7,1.0$; the other parameters are $g=0.74 \mathrm{GHz}, \Delta=4.21 \mathrm{GHz}, I_{p}=500 \mathrm{nA}$, and $\omega_{r}=8.13 \mathrm{GHz}$, the same as those previously investigated [18]. The curves with $\lambda=0.5$ (dot-dashed red line) agree perfectly with the experimental data (circle points).

to be large enough to produce an electromagnetic field characterized by a bosonic mode) controls the qubit realized by the secondary SQUID. In the limit of negligible capacitive coupling between the two SQUIDs, the circuit Hamiltonian is

$$
\begin{aligned}
\mathcal{H}_{\text {circuit }}= & \omega_{p} a^{\dagger} a-2 E_{J}^{s} \sigma^{x}-2 \tilde{L}_{p}\left(a+a^{\dagger}\right) \sigma^{x} \\
& -i M\left(a-a^{\dagger}\right) \sigma^{y},
\end{aligned}
$$

where $\omega_{p}$ is the "frequency" of the primary SQUID and $E_{J}^{s}=E_{J}^{s}\left(\phi_{\text {ext }}\right)$ provides the level splitting of the secondary SQUID, controlled by the external magnetic field; $\tilde{L}_{p}$ and $M$ are fixed by the inductance of the circuit and the mutual inductance, respectively; the gate voltage $V_{g}$ is tuned to the charge-degeneracy point. Equation (9) can be recast into the anisotropic Rabi model [17]: $\left\{\omega_{p}, E_{J}^{s}, 2 \tilde{L}_{p}, M\right\} \rightarrow\{\omega, \epsilon, g(1+\lambda), g(1-\lambda)\}$.

We comment that the implications of the simultaneous presence of the rotating and counterrotating terms have been evidenced experimentally [18-20]. The experimental system is a resonator circuit magnetically coupled to a superconducting flux qubit in the ultrastrong-coupling regime. Indeed, the experimental data are interpreted as a Bloch-Siegert energy correction of the Jaynes-Cummings dynamics. Here, we point out that the experimental results can be fitted very well in terms of our anisotropic Rabi model; see Fig. 4. (Further details are provided in Appendix B.) Such an evidence provides an indication that the inductance of the circuit is, indeed, very different from the mutual inductance between the primary and the qubit.

\section{Applications to electrons in semiconductors with spin-orbit coupling}

Spin-orbit-coupling effects have been opening up new perspectives in solid-state physics, both for fundamental research (including topological insulators and spin-Hall effects [21,22]) and applications (notably, spintronics [23]). Electronic spin-orbit (SO) coupling can be induced by the electric field acting at the two-dimensional interfaces of semiconducting heterostructure devices [23-27]. The effective Hamiltonian reads

$$
\begin{aligned}
H & =\frac{1}{2 m} \pi^{2}+\frac{1}{2} g \mu_{B} B \sigma_{z}+H_{\mathrm{SO}}, \\
H_{\mathrm{SO}} & =H_{R}+H_{D}, \\
H_{R} & =\alpha\left(\pi_{x} \sigma_{y}-\pi_{y} \sigma_{x}\right), \\
H_{D} & =\beta\left(\pi_{x} \sigma_{x}-\pi_{y} \sigma_{y}\right),
\end{aligned}
$$

where $\pi=\left\{\pi_{x}, \pi_{y}, \pi_{z}\right\}$ is the electron-canonical momentum $\pi=(\mathbf{p}-(q / c) \mathbf{A}) . H_{R}$ and $H_{D}$ are the Rashba [24] and Dresselhaus [25] spin-orbit interactions. The coupling constant $\alpha$ depends on the electric field across the well, while the Dresselhaus coupling $\beta$ is determined by the geometry of the heterostructure. The perpendicular magnetic field couples both to the electronic spin and orbital angular momentum. Applying the standard procedure that leads to the Landau levels, the Hamiltonian (10) can be recast into our anisotropic Rabi model: $\alpha=\sqrt{g^{2}+(1+\lambda)^{2}} \sin \theta$ and $\beta=\sqrt{g^{2}+(1+\lambda)^{2}} \cos \theta$. Incidentally, we observe that the simultaneous presence of Dresselhaus and Rashba contributions couples all the Landau levels, making our exact solution immediately relevant for the physics of the system.

We comment that the Hamiltonian (10) has been realized with cold fermionic atom systems, opening the avenue to study the spin-orbit effects with controllable parameters and in extremely clean environments [28-31].

\section{ENTANGLEMENT ENTROPY}

In this section, we elaborate on the phenomenon displayed in Fig. 3: For the anisotropic Rabi model, level crossings occur between eigenvalues of different parity sectors.

The crossing between the ground state and the first excited state occurs for the anisotropic case that corresponds to the exact solutions obtained by Judd [11]; see also Appendix B and Fig. 6. This phenomenon does not occur in the isotropic Rabi model and is possibly due to the competition between the rotating and counterrotating interaction terms. The position of this point can be analytically determined by the relation $K_{1}\left(x_{0}^{\text {pole }}\right)=0$ as mentioned above, i.e., $a_{0}=0$, $b_{0}=0$,

$$
g=\sqrt{\frac{2|\Delta| \omega}{1-\lambda^{2}}},
$$




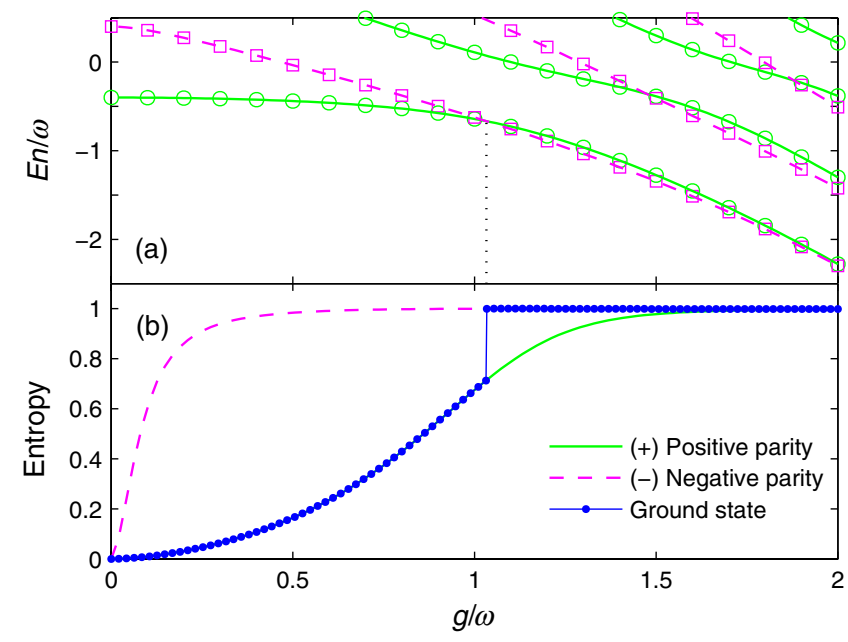

FIG. 5. Energies (upper panel) and entanglement entropies (lower panel) of the ground state and the first excited state. A level crossing occurs at $g_{c}=4 / \sqrt{15}$, marking a change of the parity of the ground state: for $g<g_{c}$, the parity is positive (solid green line), and it is negative (dashed purple line) for $g>g_{c}$, as shown in the upper panel. Correspondingly, we find that the ground-state entropy displays a sharp discontinuity at the level-crossing point, as shown in the lower panel.

$$
E_{0}=-\frac{\left(1+\lambda^{2}\right) g^{2}}{2 \omega}
$$

For the crossing of the ground state and the first excited state, we find that the series terminates at the first term, as $K_{0}=1$ and $L_{0}=2 \sqrt{\lambda} \xi^{*} /(1-\lambda)$, thereby resulting in

$$
\begin{gathered}
\phi_{1}=\frac{2 \sqrt{\lambda} \xi^{*}}{1-\lambda} \exp \left(-\frac{\sqrt{\lambda} g \xi}{\omega} z\right), \\
\phi_{2}=\exp \left(-\frac{\sqrt{\lambda} g \xi}{\omega} z\right) .
\end{gathered}
$$

Here, we study the entanglement entropy of the ground state. It can be obtained by calculating the von Neumann entropy of the spin state by tracing out the bosonic degree of freedom from the eigenstates [Eqs. (B17) and (B18)]; see Refs. [32,33] for methods. We observe that the level crossing and change of symmetry of the ground state are reflected in a clear discontinuity of the entanglement entropy (see Fig. 5). We remark that the ground states are degenerate at the level-crossing point, which is special.

\section{DISCUSSION}

In this article, we discussed a carefully chosen generalization of the Rabi model: The Hamiltonian system breaks the parity symmetry; the rotating and counterrotating interactions are governed by two different coupling constants; a further parameter introduces a phase factor in the counterrotating terms. We obtained exact energies and eigenstates of the system through the analytical properties of a transcendental function. We note that because of the anisotropic coupling, a peculiar phenomenon occurs in the energy spectrum of the system: The eigenstates belonging to different parity sectors swap in couples. We have quantified the crossing between the ground and the first excited states through the entanglement entropy of the spin system.

Our Hamiltonian systems capture the physics of notoriously important problems in different physical contexts, including a two-dimensional electron gas with a general spin-orbit interaction, a two-level atom in an electromagnetic field, and superconducting circuits in ultrastrong regimes. We explained how our results are immediately relevant for the experimental situations.

We believe that superconducting circuits made of two coupled SQUIDs could provide access to a systematic experimental study of the physical effects of the anisotropic Rabi interaction. Specifically, our study indicates that the circuit inductance, the SQUID-SQUID inductance, and the external magnetic field are the parameters that should be varied to study the crossover from the weak- to the strong-coupling regimes (see Sec. III B).

\section{ACKNOWLEDGEMENTS}

Q. T.X. and S.C. contributed equally to this work. We thank B. Englert and Wei-Bin Yan for useful discussions. We thank X.B. Zhu for discussions about the experimental realization in a superconducting flux-qubit system. This work was supported by the "973" Program (2010CB922904) and grants from the NSFC and CAS.

\section{APPENDIX A: EXACT SOLUTION OF THE ANISOTROPIC RABI MODEL}

For the Hamiltonian presented in Eq. (1), the parameter $\lambda$ controls the anisotropy between the rotating and the counterrotating terms while $\theta$ introduces only a phase into the counterrotating terms; the term $\epsilon \sigma_{x}$ breaks the $Z_{2}$ symmetry, and therefore, the eigenspace of the model [Eq. (1)] cannot be split into invariant subspaces. Nevertheless, the model [Eq. (1)] can still be solved exactly with the approach originally developed by Braak [10] for the isotropic model $\lambda=1, \theta=\epsilon=0$.

In solving exactly this model, first, for technical convenience (we comment further below), we perform a unitary transformation $U(\lambda, \theta)$ :

$$
\begin{aligned}
U(\lambda, \theta) & =\left(\begin{array}{cc}
\cos \eta e^{i \theta / 2} & -\sin \eta \\
\sin \eta & \cos \eta e^{-i \theta / 2}
\end{array}\right) \\
& =\frac{1}{\sqrt{1+\lambda}}\left(\begin{array}{cc}
\xi & -\sqrt{\lambda} \\
\sqrt{\lambda} & \xi^{*}
\end{array}\right),
\end{aligned}
$$

where $\tan \eta=\sqrt{\lambda}$ and $\xi=e^{i \theta / 2}$; when $\theta=0$ and $\xi=1$, it is a orthogonal transformation. The Hamiltonian (1) becomes 


$$
U^{\dagger} H U=\left(\begin{array}{cc}
\omega a^{\dagger} a+\sqrt{\lambda} g\left(\xi^{*} a+\xi a^{\dagger}\right)+c & \xi^{* 2}(1-\lambda) g a-d^{*} \\
\xi^{2}(1-\lambda) g a^{\dagger}-d & \omega a^{\dagger} a-\sqrt{\lambda} g\left(\xi^{*} a+\xi a^{\dagger}\right)-c
\end{array}\right),
$$

where $c=(1-\lambda) /(1+\lambda) \Delta+\left(\left(\xi+\xi^{*}\right) \sqrt{\lambda}\right) /(1+\lambda) \epsilon$ and $d=(2 \sqrt{\lambda} \xi) /(1+\lambda) \Delta-\left(\xi^{2}-\lambda\right) /(1+\lambda) \epsilon$. We exploit the Bargmann representation of bosonic operators in terms of analytic functions- $a^{\dagger} \rightarrow z$ and $a \rightarrow \partial / \partial z$-and consider the eigenfunction of the Hamiltonian as $\left(\phi_{1}, \phi_{2}\right)^{T}$; we have

$$
\begin{gathered}
{\left[\omega z \frac{d}{d z}+\sqrt{\lambda} g\left(\xi^{*} \frac{d}{d z}+\xi z\right)+c\right] \phi_{1}+\left[\xi^{* 2}(1-\lambda) g \frac{d}{d z}-d^{*}\right] \phi_{2}=E \phi_{1},} \\
{\left[\xi^{2}(1-\lambda) g z-d\right] \phi_{1}+\left[\omega z \frac{d}{d z}-\sqrt{\lambda} g\left(\xi^{*} \frac{d}{d z}+\xi z\right)-c\right] \phi_{2}=E \phi_{2} .}
\end{gathered}
$$

For convenience, we introduce the notations $\phi_{1,2}(z)=\exp (-(\sqrt{\lambda} g \xi) /(\omega) z) \psi_{1,2}(y), y=z+\sqrt{\lambda} g \xi^{*} / \omega, x=E+\lambda g^{2} / \omega$, and $f=d+(1-\lambda) \sqrt{\lambda} g^{2} \xi / \omega$. Now, we obtain

$$
\left(\omega y \frac{d}{d y}-x+c\right) \psi_{1}=\left[f^{*}-\xi^{* 2}(1-\lambda) g \frac{d}{d y}\right] \psi_{2}
$$

and

$$
\left[\left(\omega y-2 \sqrt{\lambda} g \xi^{*}\right) \frac{d}{d y}-2 \sqrt{\lambda} g \xi y+\frac{4 \lambda g^{2}}{\omega}-x-c\right] \psi_{2}=[f-(1-\lambda) g y] \psi_{1} .
$$

Assuming that the functions $\psi_{1,2}$ can be expanded as $\psi_{2}=\sum_{n=0}^{\infty} K_{n}^{+}(x) y^{n}$ and $\psi_{1}=\sum_{n=0}^{\infty} L_{n}^{+}(x) y^{n}$, from Eq. (A5), the relation between $K_{n}^{+}$and $L_{n}^{+}$is found as

$$
L_{n}^{+}=\frac{f^{*} K_{n}^{+}-\xi^{* 2}(1-\lambda) g K_{n+1}^{+}(n+1)}{n \omega-x+c} .
$$

Then, from Eq. (A6), the recursive relation of $K_{n}^{+}$is obtained:

$$
\begin{gathered}
a_{n}(x) K_{n+1}^{+}=b_{n}(x) K_{n}^{+}+c_{n}(x) K_{n-1}^{+}, \\
a_{n}(x)=\left[2 \sqrt{\lambda}-\frac{(1-\lambda) f \xi^{*}}{n \omega-x+c}\right](n+1) g \xi^{*}, \\
b_{n}(x)=\frac{4 \lambda g^{2}}{\omega}+n \omega-x-c-\frac{f^{*} f}{n \omega-x+c} \\
-\frac{(1-\lambda)^{2} g^{2} n}{(n-1) \omega-x+c}, \\
c_{n}(x)=-2 \sqrt{\lambda} g \xi+\frac{(1-\lambda) g f^{*} \xi^{2}}{(n-1) \omega-x+c},
\end{gathered}
$$

where $K_{-1}^{+}=0, K_{0}^{+}=1$, and $n=0,1,2, \ldots$ Incidentally, we comment that the unitary transformation (A1) is a key step that leads to simplifying the recursive relations that involve only three terms, as it is displayed above.
Consequently, one set of solutions is obtained:

$$
\begin{aligned}
& \phi_{1}(z)=\exp \left(-\frac{\sqrt{\lambda} g \xi}{\omega} z\right) \sum_{n=0}^{\infty} L_{n}^{+}\left(z+\frac{\sqrt{\lambda} g \xi^{*}}{\omega}\right)^{n}, \\
& \phi_{2}(z)=\exp \left(-\frac{\sqrt{\lambda} g \xi}{\omega} z\right) \sum_{n=0}^{\infty} K_{n}^{+}\left(z+\frac{\sqrt{\lambda} g \xi^{*}}{\omega}\right)^{n} .
\end{aligned}
$$

Then, substituting $z \rightarrow-z$ into Eqs. (A3) and (A4), $\phi_{1}(-z)=\bar{\phi}_{1}(z)$ and $\phi_{2}(-z)=\bar{\phi}_{2}(z)$ are eigenfunctions of the spectral problem [Eq. (A4)] as well. Such functions can be obtained by applying the same procedure that led to Eqs. (A12) and (A13). The differential equations for $\bar{\phi}_{1}(z)$ and $\bar{\phi}_{2}(z)$ are

$$
\begin{gathered}
{\left[\omega z \frac{d}{d z}-\sqrt{\lambda} g\left(\xi^{*} \frac{d}{d z}+\xi z\right)+c\right] \bar{\phi}_{1}} \\
+\left[-\xi^{* 2}(1-\lambda) g \frac{d}{d z}-d^{*}\right] \bar{\phi}_{2}=E \bar{\phi}_{1}
\end{gathered}
$$

$$
\begin{aligned}
& {\left[-\xi^{2}(1-\lambda) g z-d\right] \bar{\phi}_{1}} \\
& \quad+\left[\omega z \frac{d}{d z}+\sqrt{\lambda} g\left(\xi^{*} \frac{d}{d z}+\xi z\right)-c\right] \bar{\phi}_{2}=E \bar{\phi}_{2} .
\end{aligned}
$$


Using the following notations- $\bar{\phi}_{1,2}(z)=\exp (-(\sqrt{\lambda} g \xi) /$ $(\omega) z) \bar{\psi}_{1,2}(y), \quad y=z+\sqrt{\lambda} g \xi^{*} / \omega, \quad x=E+\lambda g^{2} / \omega, \quad$ and $\bar{f}=d-(1-\lambda) \sqrt{\lambda} g^{2} \xi / \omega$ - the above equations can be rewritten as

$$
\begin{aligned}
& {\left[\left(\omega y-2 \sqrt{\lambda} g \xi^{*}\right) \frac{d}{d y}-2 \sqrt{\lambda} g \xi y+\frac{4 \lambda g^{2}}{\omega}-x+c\right] \bar{\psi}_{1}} \\
& \quad=\left[\bar{f}^{*}+\xi^{* 2}(1-\lambda) g \frac{d}{d y}\right] \bar{\psi}_{2}, \\
& \left(\omega y \frac{d}{d y}-x-c\right) \bar{\psi}_{2}=\left[\bar{f}+\xi^{2}(1-\lambda) g y\right] \bar{\psi}_{1} .
\end{aligned}
$$

Expanding the functions $\bar{\psi}_{1,2}$ as $\bar{\psi}_{1}=\sum_{n=0}^{\infty} K_{n}^{-}(x) y^{n}$ and $\bar{\psi}_{2}=\sum_{n=0}^{\infty} L_{n}^{-}(x) y^{n}$, from Eq. (A17), we find the relation of $K_{n}^{-}$and $L_{n}^{-}$:

$$
L_{n}^{-}=\frac{\bar{f} K_{n}^{-}+\xi^{2}(1-\lambda) g K_{n-1}^{-}}{n \omega-x-c} .
$$

Then, from Eq. (A16), we obtain the recursive relation

$$
\begin{gathered}
\bar{a}_{n}(x) K_{n+1}^{-}=\bar{b}_{n}(x) K_{n}^{-}+\bar{c}_{n}(x) K_{n-1}^{-}, \\
\bar{a}_{n}(x)=\left[2 \sqrt{\lambda}+\frac{(1-\lambda) \bar{f} \xi^{*}}{(n+1) \omega-x-c}\right](n+1) g \xi^{*}, \\
\bar{b}_{n}(x)=\frac{4 \lambda g^{2}}{\omega}+n \omega-x+c-\frac{\bar{f}^{*} \bar{f}}{n \omega-x-c} \\
-\frac{(1-\lambda)^{2} g^{2}(n+1)}{(n+1) \omega-x-c} \\
\bar{c}_{n}(x)=-2 \sqrt{\lambda} g \xi-\frac{(1-\lambda) g \bar{f}^{*} \xi^{2}}{n \omega-x-c},
\end{gathered}
$$

where $K_{-1}^{-}=0, K_{0}^{-}=1$, and $n=0,1,2, \ldots$.

Going back to the original notations, we have

$$
\begin{aligned}
& \bar{\phi}_{1}(z)=\exp \left(-\frac{\sqrt{\lambda} g \xi}{\omega} z\right) \sum_{n=0}^{\infty} K_{n}^{-}\left(z+\frac{\sqrt{\lambda} g \xi^{*}}{\omega}\right)^{n}, \\
& \bar{\phi}_{2}(z)=\exp \left(-\frac{\sqrt{\lambda} g \xi}{\omega} z\right) \sum_{n=0}^{\infty} L_{n}^{-}\left(z+\frac{\sqrt{\lambda} g \xi^{*}}{\omega}\right)^{n} .
\end{aligned}
$$

Considering the relation of these two sets of eigenstates mentioned above- $\phi_{1}(-z)=C \bar{\phi}_{1}(z)$ and $\phi_{2}(-z)=$ $C \bar{\phi}_{2}(z)$-and then canceling the arbitrary constant $C$, a transcendental function can be constructed as

$$
G_{\epsilon}(x ; z)=\phi_{1} \bar{\phi}_{2}-\phi_{2} \bar{\phi}_{1}
$$

Because $G_{\epsilon}(x ; z)$ is well defined at $z= \pm \sqrt{\lambda} g \xi^{*} / \omega$ within the convergent radius $R=2 \sqrt{\lambda} g \xi^{*} / \omega$, we can set $z=0$
[10]. The function $G_{\epsilon}(x ; 0)$ is analytic in the complex plane, except in the simple poles

$$
\begin{array}{r}
x_{n}^{\mathrm{pole}}=n \omega-\frac{(1-\lambda)^{2} g^{2}}{2 \omega}+\frac{\left(\xi+\lambda \xi^{*}\right) \epsilon}{2 \sqrt{\lambda}}, \\
\bar{x}_{n}^{\mathrm{pole}}=(n+1) \omega-\frac{(1-\lambda)^{2} g^{2}}{2 \omega}-\frac{\left(\xi+\lambda \xi^{*}\right) \epsilon}{2 \sqrt{\lambda}},
\end{array}
$$

which follows from the $0 \mathrm{~s}$ of the denominator of $K_{n}^{ \pm}$: $a_{n}(x)=0$ and $\bar{a}_{n}(x)=0$ in Eqs. (A8) and (A19), respectively. Then, the eigenvalues and eigenstates can be obtained by solving $G_{\epsilon}(x)=0$ :

$$
\begin{gathered}
E_{n}=x_{n}-\frac{\lambda g^{2}}{\omega}, \\
\Psi_{n}=U(\lambda, \theta)\left(\begin{array}{c}
\phi_{1}\left(x_{n}\right) \\
\phi_{2}\left(x_{n}\right)
\end{array}\right) \\
=U(\lambda, \theta)\left(\begin{array}{c}
\left.\sum_{n=0}^{\infty} L_{n}^{+}|n\rangle\right\rangle \\
\left.\sum_{n=0}^{\infty} K_{n}^{+}|n\rangle\right\rangle
\end{array}\right) .
\end{gathered}
$$

Using the second solution, the eigenstates of the Hamiltonian with $a \rightarrow-a$ and $a^{\dagger} \rightarrow-a^{\dagger}$ can be obtained:

$$
\begin{aligned}
\bar{\Psi}_{n} & =U(\lambda, \theta)\left(\begin{array}{l}
\bar{\phi}_{1}\left(x_{n}\right) \\
\bar{\phi}_{2}\left(x_{n}\right)
\end{array}\right) \\
& =U(\lambda, \theta)\left(\begin{array}{c}
\left.\sum_{n=0}^{\infty} K_{n}^{-}|n\rangle\right\rangle \\
\left.\sum_{n=0}^{\infty} L_{n}^{-}|n\rangle\right\rangle
\end{array}\right),
\end{aligned}
$$

where

$$
\begin{aligned}
& |n\rangle\rangle \doteq\left(a^{\dagger}+\frac{\sqrt{\lambda} g \xi^{*}}{\omega}\right)^{n}\left|-\frac{\sqrt{\lambda} g \xi}{\omega}\right\rangle, \\
& \left|-\frac{\sqrt{\lambda} g \xi}{\omega}\right\rangle=e^{-\left(\lambda g^{2} / 2 \omega^{2}\right)-(\sqrt{\lambda} g \xi / \omega) a^{\dagger}}|0\rangle .
\end{aligned}
$$

In the case of $\lambda=1$ and $\theta=0$, we can recover the results given by Braak [10] and some generalized results [34-36]. Some other detailed calculations can be found in Appendix B.

\section{APPENDIX B: RESULTS FOR THE $Z_{2}$ SYMMETRIC CASE}

For $\epsilon=0$, the anisotropic Rabi model enjoys a $Z_{2}$ symmetry that reflects the conservation of the parity of the operator

$$
\hat{N}=a^{\dagger} a+\frac{1}{2}\left(\sigma_{z}+1\right) .
$$


In this case, the phase factors $e^{ \pm i \theta}$ in the Hamiltonian can be canceled by a unitary transformation $R(\theta)$ :

$$
\begin{aligned}
& R(\theta)=e^{i(\theta / 2)[\hat{N}-(1 / 2)]}=e^{i(\theta / 2)\left[\left(\sigma_{z} / 2\right)+a^{\dagger} a\right]}, \\
& R^{\dagger}(\theta) H R(\theta)= \omega a^{\dagger} a+\Delta \sigma_{z}+g\left[\sigma^{+} a+\sigma^{-} a^{\dagger}\right. \\
&\left.+\lambda\left(\sigma^{+} a^{\dagger}+\sigma^{-} a\right)\right] .
\end{aligned}
$$

Therefore, the parameter $\theta$ gives no contribution to the energy spectra but enters only the wave functions.

We shall see that the $Z_{2}$ symmetry effectively simplifies the procedure of finding the exact spectrum since the transcendental function $G_{\epsilon}(x), \epsilon=0$, can be discussed separately in the different parity sectors.

To simplify the solution of the spectral problem, we resort to a similar trick we employed above. Namely, we apply the rotation

$$
\begin{aligned}
V & =U^{\dagger}(\lambda, \theta) W \\
& =\frac{1}{\sqrt{2(1+\lambda)}}\left(\begin{array}{cc}
\xi^{*}+\sqrt{\lambda} & -\xi^{*}+\sqrt{\lambda} \\
\xi-\sqrt{\lambda} & \xi+\sqrt{\lambda}
\end{array}\right),
\end{aligned}
$$

with

$$
W=\frac{1}{\sqrt{2}}\left(\begin{array}{cc}
1 & -1 \\
1 & 1
\end{array}\right)
$$

to the Hamiltonian (B3). Now, we have the Hamiltonian

$$
W^{\dagger} H W=\left(\begin{array}{cc}
\omega a^{\dagger} a+\frac{1+\lambda}{2}\left(a+a^{\dagger}\right) & \frac{1-\lambda}{2}\left(a-a^{\dagger}\right)+\Delta \\
-\frac{1-\lambda}{2}\left(a-a^{\dagger}\right)+\Delta & \omega a^{\dagger} a-\frac{1+\lambda}{2}\left(a+a^{\dagger}\right)
\end{array}\right) .
$$

The eigenfunctions $\phi_{1}$ and $\phi_{2}$ (and similarly $\bar{\phi}_{1}$ and $\bar{\phi}_{2}$ ) in the main text transform according to $\left(\varphi_{1}, \varphi_{2}\right)^{T}=V^{\dagger}\left(\phi_{1}, \phi_{2}\right)^{T}$ :

$$
\begin{gathered}
\varphi_{1}=\frac{(\xi+\sqrt{\lambda}) \phi_{1}+\left(\xi^{*}-\sqrt{\lambda}\right) \phi_{2}}{\sqrt{2(1+\lambda)}}, \\
\varphi_{2}=\frac{(-\xi+\sqrt{\lambda}) \phi_{1}+\left(\xi^{*}+\sqrt{\lambda}\right) \phi_{2}}{\sqrt{2(1+\lambda)}} .
\end{gathered}
$$

We know that $\phi_{1}$ and $\phi_{2}$ read as

$$
\begin{aligned}
& \phi_{1}(z)=\exp \left(-\frac{\sqrt{\lambda} g \xi}{\omega} z\right) \sum_{n=0}^{\infty} L_{n}(x)\left(z+\frac{\sqrt{\lambda} g \xi^{*}}{\omega}\right)^{n}, \\
& \phi_{2}(z)=\exp \left(-\frac{\sqrt{\lambda} g \xi}{\omega} z\right) \sum_{n=0}^{\infty} K_{n}(x)\left(z+\frac{\sqrt{\lambda} g \xi^{*}}{\omega}\right)^{n},
\end{aligned}
$$

where

$$
L_{n}=\frac{f^{*} K_{n}-\xi^{* 2}(1-\lambda) g K_{n+1}(n+1)}{n \omega-x+c} .
$$

Here, the + superindices are omitted.

The $Z_{2}$ symmetry reflects into a symmetry in the eigenfunction $\varphi_{2}(-z)=C \varphi_{1}(z)$, where $C$ is an arbitrary constant. Without loss of generality, we take $\varphi_{1,2}$ as normalized and real. In this case, $C= \pm 1$, and the transcendental function $G$ is

$$
G_{ \pm}^{\lambda}(x ; z)=\varphi_{2}(-z) \mp \varphi_{1}(z)=0, \quad \forall z \in \mathcal{C} .
$$

Setting $z=0$ as the above section, and substituting $\varphi_{1,2}$ by $\phi_{1,2}$,

$$
\begin{aligned}
& G_{+}^{\lambda}(x)=-\xi \phi_{1}+\sqrt{\lambda} \phi_{2}, \\
& G_{-}^{\lambda}(x)=\sqrt{\lambda} \phi_{1}+\xi^{*} \phi_{2} .
\end{aligned}
$$

The energy spectrum can be divided into two cases. One case is the regular solution, which is solely determined by $0 \mathrm{~s}$ of the transcendental function. Another case corresponds to the exceptional solutions. For this case, we can consider first the the poles of the transcendental function determined by setting $a_{n}(x)=0$ :

$$
x_{n}^{\text {pole }}=n \omega-\frac{(1-\lambda)^{2} g^{2}}{2 \omega} .
$$

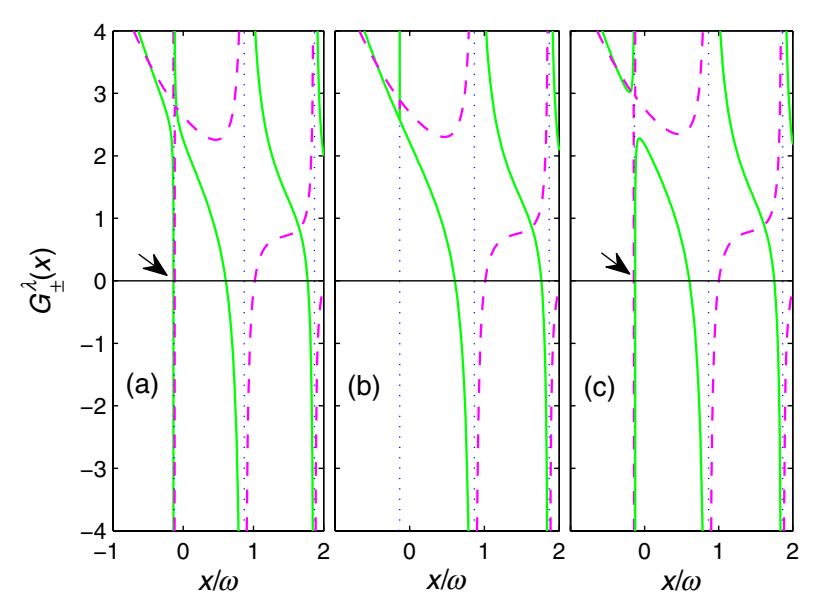

FIG. 6. Comparison of transcendental functions near the ground-state degenerate point. From left to right, the parameters are (a) $g=g_{c}-0.01$, (b) $g=g_{c}$, and (c) $g=g_{c}+0.01$, where $g_{c}=4 / \sqrt{15}$, which is the degenerate point. $G_{+}^{\lambda}(x)$ (solid green line) and $G_{-}^{\lambda}(x)$ (dashed purple line) are presented as functions of $x$, where $\omega=1, \Delta=0.4, \lambda=0.5$, and $\theta=0$. The differences between (a)-(c) can be observed, for example, at the points marked by arrows in the figures. For (a), at the point marked by an arrow, the energy of positive parity is slightly less than the energy of negative parity; it is reversed in (c), while in (b), the pole is lifted since $K_{1}=0$. 
At the same time, if $K_{n+1}\left(x_{n}^{\text {pole }}\right)=0$ for special values of the parameters $g$ and $\Delta$, the poles can be lifted because the numerator of $G_{ \pm}^{\lambda}$ is also vanishing. Figure 6 shows the transition between regular solutions to exceptional solutions. These special solutions are Judd-type solutions for the anisotropic Rabi model, corresponding to the so-called isolated integrability [11]. Owing to $G_{ \pm}^{\lambda} \neq 0$, these eigenvalues have no definite parity, and a double degeneracy of the eigenvalues occurs [see Fig. 3(b) in the main text]. In this case, the infinite series solutions $K_{n}$ and $L_{n}$ can be terminated as finite series solutions. We note that, in particular, there is no crossing with the same parity. Incidentally, a crossing between the ground state and the first excited state occurs for the anisotropic case that corresponds to the exact solutions obtained by Judd [11], as already shown in Sec. IV.

Figure 7 shows that the parity of the ground state changes sign when passing through the level-crossing point. When $g / \omega$ is small, the ground state is positive parity and the first excited state is negative parity; after passing through the crossing point where the parity changes sign, the ground state is negative parity and the first excited state is positive parity. This parity changing can be demonstrated by the intrinsic symmetries of the ground state.

The ground state of Hamiltonian (1) with vanishing $\epsilon$ can be written as

$$
\Psi(z)=\left(\begin{array}{c}
\varphi_{1}(z) \\
\varphi_{2}(z)
\end{array}\right)
$$

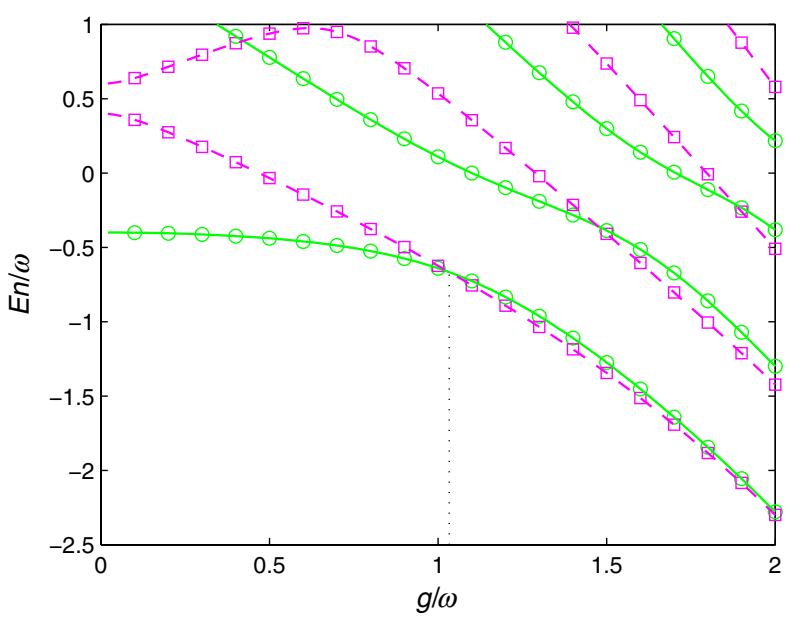

FIG. 7. Ground-state level crossing. The ground-state energy and the first-excited-state energy are crossed for the anisotropic Rabi model with $Z_{2}$ symmetry. The parity of the ground state changes when passing through the crossing point. Here, $\epsilon=0$ for keeping $Z_{2}$ symmetry; we choose $\Delta=0.4$ and $\lambda=0.5$, so $g=$ $4 / \sqrt{15}$ and $E_{0}^{e}=-(2 / 3)$ due to Eqs. (11) and (12). The solid green lines are for positive parity, and the dashed purple lines are for negative parity. The circles and blocks are numerical data; they agree well with the analytical results shown in the lines. Part of this figure is shown in the upper panel of Fig. 5. or $\varphi_{1}(z) \rightarrow \varphi_{2}(-z)$ and $\varphi_{2}(z) \rightarrow \varphi_{1}(-z)$ because of the $Z_{2}$ symmetry. Additionally, when $g / \omega$ is small, which is less than the crossing-point value, the ground state is parity positive and can be simplified as

$$
\Psi_{+}(z)=\left(\begin{array}{c}
\varphi_{1}(z) \\
\varphi_{1}(-z)
\end{array}\right)
$$

In comparison, when $g / \omega$ is larger than the crossing-point value, the ground state is parity negative and takes the form

$$
\Psi_{-}(z)=\left(\begin{array}{c}
\varphi_{1}(z) \\
-\varphi_{1}(-z)
\end{array}\right) .
$$

However, we find that both $\Psi_{ \pm}(z)$ are not the eigenstates of the Hamiltonian at the ground-state level-crossing point.

We may reformulate the ground state with different parities as

$$
\begin{aligned}
& \Psi_{+}(z)=\left(\begin{array}{c}
\varphi_{1}(z)+\varphi_{2}(-z) \\
\varphi_{2}(z)+\varphi_{1}(-z)
\end{array}\right), \\
& \Psi_{-}(z)=\left(\begin{array}{c}
\varphi_{1}(z)-\varphi_{2}(-z) \\
\varphi_{2}(z)-\varphi_{1}(-z)
\end{array}\right) .
\end{aligned}
$$

Those two states are the ground state and the first excited state. They are the correct eigenstates that correspond to different parities in the whole region, including the levelcrossing point. Explicitly, one may confirm that $\Psi_{ \pm}(z)$ in Eqs. (B17) and (B18) are similar to the results in Eqs. (B15) and (B16) when $g / \omega$ is not at the crossing point, respectively. The ground energy degeneracy at the crossing point also implies that arbitrary linear combinations of states $\Psi_{+}(z)$ and $\Psi_{-}(z)$ in Eqs. (B17) and (B18) are also the ground-state eigenstates.

With the help of the solutions [Eqs. (13) and (14)] and also considering the transformation (B5), the eigenstates at the level-crossing point can be written as follows, up to a whole factor

$W \Psi_{+}(z)=\left(\begin{array}{c}\sqrt{\lambda}\left[\exp \left(-\frac{\sqrt{\lambda} g \xi}{\omega} z\right)-\exp \left(\frac{\sqrt{\lambda} g \xi}{\omega} z\right)\right] \\ \xi^{\dagger}\left[\exp \left(-\frac{\sqrt{\lambda} g \xi}{\omega} z\right)+\exp \left(\frac{\sqrt{\lambda} g \xi}{\omega} z\right)\right]\end{array}\right)$,

$W \Psi_{+}(z)=\left(\begin{array}{c}\sqrt{\lambda}\left[\exp \left(-\frac{\sqrt{\lambda} g \xi}{\omega} z\right)+\exp \left(\frac{\sqrt{\lambda} g \xi}{\omega} z\right)\right] \\ \xi^{\dagger}\left[\exp \left(-\frac{\sqrt{\lambda} g \xi}{\omega} z\right)-\exp \left(\frac{\sqrt{\lambda} g \xi}{\omega} z\right)\right]\end{array}\right)$.

We remark that because of the ground-state energy degeneracy, the linear combinations of the ground states may lead to simpler solutions 


$$
\Psi(z)=\left(\begin{array}{c}
\sqrt{\lambda} \exp \left(-\frac{\sqrt{\lambda} g}{\omega} z\right) \\
\exp \left(-\frac{\sqrt{\lambda} g}{\omega} z\right)
\end{array}\right) ; \quad\left(\begin{array}{c}
\sqrt{\lambda} \exp \left(\frac{\sqrt{\lambda} g}{\omega} z\right) \\
-\exp \left(\frac{\sqrt{\lambda} g}{\omega} z\right)
\end{array}\right) .
$$

It can be checked that these the above expression for $\Psi(z)$ provides the eigenstates of the Hamiltonian (1) for vanishing $\epsilon=0$ and $\theta=0$, with degeneracy conditions [Eqs. (11) and (12)].

Finally, we remark that the Bargmann representation of the bosonic operator used in our model can also be used in the JC model, which has $U(1)$ symmetry. The eigenvalues and eigenstates can be obtained in the familiar steps but are simpler because the total number $\hat{N}=a^{\dagger} a+1 / 2\left(\sigma_{z}+1\right)$ is conserved.

\section{Fitting with experimental data for the superconducting circuits in the strongly coupled regime}

We consider the energy gaps between the anisotropic Rabi model and the JC model. Such energy differences, generalizing the Bloch-Siegert effect of the isotropic Rabi model, play important roles in many physical applications where the strong-coupling regime is the actual one, like in the superconducting circuits and some similar physical systems [37-43].

Ordinarily, there is no general form for the gap, but we can analyze it at the degenerate points. The ground-state energy of the JC model is $E_{0}^{\mathrm{JC}}=-\Delta$, so the ground-state gap at $|\Delta|=\left(1-\lambda^{2}\right) g^{2} / 2 \omega$ is

$$
\Delta E_{0}=-\Delta-E_{0}^{e}=\frac{\lambda^{2} g^{2}}{\omega}
$$

when $\lambda=0$ (the JC limit), the gap vanishes; for $\lambda=1$, the gap is just the standard Bloch-Siegert shift in the Rabi model $\Delta E_{0}=g^{2} / \omega$. For $\lambda \neq 1$, the first-excited-state energy gap with the $\mathrm{JC}$ is

$$
\Delta E_{1}=\frac{\omega}{2}-\sqrt{\left(\Delta-\frac{\omega}{2}\right)^{2}+g^{2}}+\frac{\left(1+\lambda^{2}\right) g^{2}}{2 \omega} .
$$

For small $g / \omega, \Delta E_{1} \approx \lambda^{2} g^{4} / \omega^{3}$, remarkably different from the standard Bloch-Siegert shift $g^{2} / \omega$.

In the Rabi model, there is a crossing between the second and the third energy levels at $|\Delta|=\sqrt{\omega^{2}-4 g^{2}}, E=\omega-$ $g^{2} / \omega$ [10]; the second energy level of the JC model in small $g / \omega$ can be written as

$$
\begin{aligned}
E_{2}^{\mathrm{JC}} & =\frac{\omega}{2}+\sqrt{\left(\Delta-\frac{\omega}{2}\right)^{2}+g^{2}} \\
& \approx \omega-\frac{g^{2}}{\omega}+\frac{g^{4}}{\omega^{3}} .
\end{aligned}
$$

Obviously, the second excited energy difference between the JC model and the Rabi model is in the $g^{4} / \omega^{3}$ scale, too. However, we compare the third excited energy difference at this point as

$$
\begin{aligned}
E_{3}^{\mathrm{JC}}-E_{1}^{e, \mathrm{Rabi}} & =\frac{3 \omega}{2}-\sqrt{\left(\Delta-\frac{\omega}{2}\right)^{2}+2 g^{2}}-\left(\omega-\frac{g^{2}}{\omega}\right) \\
& \approx \frac{g^{2}}{\omega}-\frac{2 g^{4}}{\omega^{3}}
\end{aligned}
$$

where the condition $g / \omega \ll 1$ is used; the difference is still in the $g^{2} / \omega$ scale. Maybe, the energy differences of the second and third excited states are strange at the degenerate point. Those differences may be verified by the recent experiments with ultrastrong coupling.

In the ultrastrong-coupling regime, the deviation from the JC model known as the Bloch-Siegert shift was experimentally observed [18], which is a resonator circuit resonator magnetically coupled to a superconducting flux qubit in the ultrastrong-coupling regime, and the system can be modeled by the Hamiltonian

$$
\begin{aligned}
H^{\prime}= & \frac{\omega_{q}}{2}\left(\sigma_{z}+1\right)+\omega_{r} a^{\dagger} a \\
& +g\left(\cos \vartheta \sigma_{z}-\sin \vartheta \sigma_{x}\right)\left(a+a^{\dagger}\right),
\end{aligned}
$$

with $\omega_{q} \equiv \sqrt{\epsilon^{2}+\Delta^{2}}, \epsilon=2 \pi I_{p}\left(\Phi-\Phi_{0} / 2\right)$, and $\tan \vartheta=\Delta / \epsilon$, where $\hbar$ is conventionally set to 1 .

Following the results shown in Ref. [18], if we neglect the term $g \cos \vartheta \sigma_{z}\left(a+a^{\dagger}\right)$, which only contributes a constant $-g^{2} \cos ^{2} \vartheta / \omega_{r}$ to the second order under the transformation $U=\exp \left[-g \cos \vartheta / \omega_{r} \sigma_{z}\left(a-a^{\dagger}\right)\right]$, and by omitting the counterrotating term, the corresponding JC model is given by

$H_{\mathrm{JC}}=\frac{\omega_{q}}{2}\left(\sigma_{z}+1\right)+\omega_{r} a^{\dagger} a-g \sin \vartheta\left(\sigma^{-} a^{\dagger}+\sigma^{+} a\right)$.

In the ultrastrong-coupling regime, the rotating-wave approximation is thus inappropriate; the experimental results of this system will not agree with the JC model. The Bloch-Siegert shift caused by the counterrotating term is evidently observed.

Then, we directly use our proposed anisotropic model to fit this system:

$$
\begin{aligned}
H_{a-\mathrm{Rabi}}= & \frac{\omega_{q}}{2}\left(\sigma_{z}+1\right)+\omega_{r} a^{\dagger} a \\
& -g \sin \vartheta\left[\sigma^{-} a^{\dagger}+\sigma^{+} a+\lambda\left(\sigma^{-} a+\sigma^{+} a^{\dagger}\right)\right],
\end{aligned}
$$

where the anisotropic parameter $\lambda$ is decided by fitting, and $g=0.74 \mathrm{GHz}, \Delta=4.21 \mathrm{GHz}, I_{p}=500 \mathrm{nA}$, and $\omega_{r}=$ $8.13 \mathrm{GHz}$ are the same as those previously obtained [18]. As shown in Fig. 4, we can find that the experimental data 
agree perfectly with the case $\lambda=0.5$ (dot-dashed red line), which is neither the Rabi model nor the JC model, and we remark that the result of the $\lambda=0.7$ case seems similar to the Hamiltonian (B26) (solid black line), and the $\lambda=0$ case is the same as the JC Hamiltonian (B27) (dashed black line). Here, we try to comment that by this experimental setup, the anisotropic Rabi model may be tested in a full regime by using qubit devices with strength of coupling ranging from weak to ultrastrong up to $g \approx 2 \mathrm{GHz}$ within current technologies.

[1] I. I. Rabi, Space Quantization in a Gyrating Magnetic Field, Phys. Rev. 51, 652 (1937).

[2] M.O. Scully and M.S. Zubairy, Quantum Optics (Cambridge University Press, Cambridge, England, 1997).

[3] M. Wagner, Unitary Transformations in Solid State Physics (North-Holland, Amsterdam, 1986).

[4] E. T. Jaynes and W. W. Cummings, Comparison of Quantum and Semiclassical Radiation Theories with Application to the Beam Maser, Proc. IEEE 51, 89 (1963).

[5] J. M. Raimond, M. Brune, and S. Haroche, Manipulating Quantum Entanglement with Atoms and Photons in a Cavity, Rev. Mod. Phys. 73, 565 (2001).

[6] D. Liebfried, R. Blatt, C. Monroe, and D. Wineland, Quantum Dynamics of Single Trapped Ions, Rev. Mod. Phys. 75, 281 (2003).

[7] D. Englund, A. Faraon, I. Fushman, N. Stoltz, P. Petroff, and J. Vučković, Controlling Cavity Reflectivity with a Single Quantum Dot, Nature (London) 450, 857 (2007).

[8] E. K. Irish, Generalized Rotating-Wave Approximation for Arbitrarily Large Coupling, Phys. Rev. Lett. 99, 173601 (2007).

[9] L. Amico, H. Frahm, A. Osterloh, and G. A. P. Ribeiro, Integrable Spin-Boson Models Descending from Rational Six-Vertex Models, Nucl. Phys. B787, 283 (2007).

[10] D. Braak, Integrability of the Rabi Model, Phys. Rev. Lett. 107, 100401 (2011).

[11] B. R. Judd, Exact Solutions to a Class of Jahn-Teller Systems, J. Phys. C 12, 1685 (1979).

[12] F. Bloch and A. Siegert, Magnetic Resonance for Nonrotating Fields, Phys. Rev. 57, 522 (1940).

[13] J. H. Shirley, Solution of the Schrödinger Equation with a Hamiltonian Periodic in Time, Phys. Rev. 138, B979 (1965).

[14] D. Esteve, in Superconducting Qubits, Proceedings of the Les Houches Summer School, Session LXXIX, edited by D. Esteve and J.-M. Raimond (Elsevier, Amsterdam, 2004).

[15] I. Chiorescu, P. Bertet, K. Semba, Y. Nakamura, C. J. P. M. Harmans, and J. E. Mooij, Coherent Dynamics of a Flux Qubit Coupled to a Harmonic Oscillator, Nature (London) 431, 159 (2004).

[16] K. V. R. M. Murali, Z. Dutton, W. Oliver, D. Crankshaw, and T. Orlando, Probing Decoherence with Electromagnetically Induced Transparency in Superconductive Quantum Circuits, Phys. Rev. Lett. 93, 087003 (2004).
[17] L. Amico and K. Hikami, Integrable Spin-Boson Interaction in the Tavis-Cummings Model from a General Boundary Twist, Eur. Phys. J. B 43, 387 (2005).

[18] P. Forn-Diaz, J. Lisenfeld, D. Marcos, J. J. García-Ripoll, E. Solano, C. J. P. M. Harmans, and J. E. Mooij, Observation of the Bloch-Siegert Shift in a Qubit-Oscillator System in the Ultrastrong Coupling Regime, Phys. Rev. Lett. 105, 237001 (2010).

[19] T. Niemczyk et al., Circuit Quantum Electrodynamics in the Ultrastrong-Coupling Regime, Nat. Phys. 6, 772 (2010).

[20] J. Tuorila, M. Silveri, M. Sillanpää, E. Thuneberg, Y. Makhlin, and P. Hakonen, Stark Effect and Generalized Bloch-Siegert Shift in a Strongly Driven Two-Level System, Phys. Rev. Lett. 105, 257003 (2010).

[21] M.Z. Hasan and C. L. Kane, Colloquium: Topological Insulators, Rev. Mod. Phys. 82, 3045 (2010).

[22] Y. K. Kato, R. C. Myers, A. C. Gossard, and D. D. Awschalom, Observation of the Spin Hall Effect in Semiconductors, Science 306, 1910 (2004).

[23] S. Datta and B. Das, Electronic Analog of the Electro-optic Modulator, Appl. Phys. Lett. 56, 665 (1990).

[24] E. I. Rashba, Properties of Semiconductors with an Extremum Loop. 1. Cyclotron and Combinational Resonance in a Magnetic Field Perpendicular to the Plane of the Loop, Sov. Phys. Solid State 2, 1109 (1960).

[25] G. Dresselhaus, Spin-Orbit Coupling Effects in Zinc Blende Structures, Phys. Rev. 100, 580 (1955).

[26] J. Schliemann, J. Carlos Egues, and D. Loss, Variational Study at the $\nu=1$ Quantum Hall Ferromagnet in the Presence of Spin-Orbit Interaction, Phys. Rev. B 67, 085302 (2003).

[27] L. W. Molenkamp, G. Schmidt, and G. E. W. Bauer, Rashba Hamiltonian and Electron Transport, Phys. Rev. B 64, 121202 (2001).

[28] Y. J. Lin, K. Jiménez-García, and I. B. Spielman, Spin-Orbit-Coupled Bose-Einstein Condensates, Nature (London) 471, 83 (2011).

[29] X. J. Liu, M. F. Borunda, X. Liu, and J. Sinova, Effect of Induced Spin-Orbit Coupling for Atoms via Laser Fields, Phys. Rev. Lett. 102, 046402 (2009).

[30] C. J. Kennedy, G. A. Siviloglou, H. Miyake, W. C. Burton, and W. Ketterle, Spin-Orbit Coupling and Spin Hall Effect for Neutral Atoms without Spin-Flips, Phys. Rev. Lett. 111, 225301 (2013).

[31] I. Bloch, J. Balibard, and S. Nascimbène, Quantum Simulations with Ultracold Quantum Gases, Nat. Phys. 8, 267 (2012).

[32] L. Amico, R. Fazio, A. Osterloh, and V. Vedral, Entanglement in Many-Body Systems, Rev. Mod. Phys. 80, 517 (2008).

[33] J. Eisert, M. Cramer, and M. B. Plenio, Colloquium: Area Law for the Entanglement Entropy, Rev. Mod. Phys. 82, 277 (2010).

[34] V. V. Albert, Quantum Rabi Model for N-State Atoms, Phys. Rev. Lett. 108, 180401 (2012).

[35] Q. H. Chen, C. Wang, S. He, T. Liu, and K.-L. Wang, Exact Solvability of the Quantum Rabi Model Using Bogoliubov Operators, Phys. Rev. A 86, 023822 (2012). 
[36] M. Tomka, O. El Araby, M. Pletyukhov, and V. Gritsev, Exceptional and Regular Spectra of the Generalized Rabi Model, arXiv:1307.7876.

[37] J.E. Mooij et al., Josephson Persistent-Current Qubit, Science 285, 1036 (1999).

[38] G. Günter et al., Sub-cycle Switch-On of Ultrastrong Light-Matter Interaction, Nature (London) 458, 178 (2009).

[39] A. Waltraff, D. I. Schuster, A. Blais, L. Frunzio, R.- S. Huang, J. Majer, S. Kumar, S. M. Girvin, and R. J. Schoelkopf, Strong Coupling of a Single Photo to a Superconducting Qubit Using Circuit Quantum Electrodynamics, Nature (London) 431, 162 (2004).
[40] D. I. Schuster et al., Resolving Photon Number States in a Superconducting Circuit, Nature (London) 445, 515 (2007).

[41] B. Peropadre, P. Forn-Díaz, E. Solano, and J. J. GarcíaRipoll, Switchable Ultrastrong Coupling in Circuit QED, Phys. Rev. Lett. 105, 023601 (2010).

[42] Y. Nakamura, Yu. A. Pashkin, and J. S. Tsai, Rabi Oscillations in a Josephson-Junction Charge Two-Level System, Phys. Rev. Lett. 87, 246601 (2001).

[43] X. B. Zhu et al., Coherent Coupling of a Superconducting Flux Qubit to an Electron Spin Ensemble in Diamond, Nature (London) 478, 221 (2011). 